\title{
CME quiz
}

The purpose of this quiz is to provide a convenient means of self-assessment of your reading of the scientific content of this issue of JAOA. Enter your answers to the questions in the spaces provided so that you can easily check them with the answers that will be published next month.

To apply for CME credit, transfer your answers to the mailin card on page $\mathbf{7 7}$ and return it to the CME office. So that you may complete this self-assessment in privacy, use only your member number to apply for CME credit. The CME office will record only the fact that you have completed the self-assessment test. Any grading will be done by the Editorial Department only for the purpose of planning areas of study which may be helpful to cover in future issues of $J A O A$.

1. When a heel lift was used by Irvin to reduce mild lumbar scoliosis in adults, the final thickness of the lift was determined according to which of the following?

(a) Uneven heights of the femoral heads. (b) Unlevel heights of the iliac crests.

(c) Unlevelness of the sacral base.

(d) Disparity of leg lengths.

(e) Degree of scoliosis.

2. Irvin found that the reversibility of mild lumbar scoliosis in adults by leveling the sacral base is which of the following?

(a) None.

(b) One fifth.

(c) One fourth.

(d) One third.

(e) One half.

3. Which of the following statements regarding paroxysmal supraventricular tachycardia in pregnancy is true?

(a) Those measures that are effective in the general population can be safely used in the pregnant patient under monitored conditions.

(b) Hemodynamic compromise usually warrants electric cardioversion in a closely monitored setting.

(c) The initial treatment should begin with vagal maneuvers fol- lowed by pharmacologic therapy, including verapamil, $\beta$-blockers, digoxin, or type 1a antiarrhythmic agents.

(d) None of the above is true.

(e) All of the above are true.

4. Which of the following predisposing clinical conditions are associated with Vibrio vulnificus septicemia?

(a) Liver disease.

(b) Leukemia.

(c) Diabetes mellitus.

(d) Hemochromatosis.

(e) Steroid-dependent asthma.

(f) Peptic ulcer disease.

(g) Chronic renal failure.

(h) All of the above.

5. If in the initial presentation of Vibrio vulnificus primary septicemia refractory hypotension is present, the mortality is $100 \%$.

(a) True.

(b) False. 